\title{
Solar System Cartography for High School Education: A Dual Scale Approach
}

\author{
Stephan Wondrak ${ }^{\mathrm{a}}$ \\ ${ }^{a}$ Swiss Federal Statistical Office, stephan@wondrak.ch
}

Keywords: Solar System, Planets, Orbits, Web Application, 2D-Visualisation

\begin{abstract}
:
This presentation deals with certain aspects of Solar System Cartography, in relation to the development of a web application for high school education. The Planet Tool is introduced, a web application for the visualisation of physical and orbital characteristics of the planets in our Solar System ${ }^{1}$. A main principle of the Planet Tool is the reduction of the presented data down to the essential information, like it is also done in the process of map editing. The capabilities of client-side web technologies are used for the generation of interactive 2-dimensional vector graphics.

A web page shows the orbits and the current positions of the planets in a heliocentric Cartesian coordinate system, projected into the ecliptic plane. The planets and the Sun have an equal size and are visible as small white dots. Hence, only the orbits have a consistent map scale. The uniform map symbols for the planets and the Sun were placed without the consideration of the resultant, different scale number for each celestial body. However, the true size of the planets and the Sun can be shown with two scaling options:
\end{abstract}

1. The planets and the Sun are shown in the scale of the orbits. A strong zoom to a planet is necessary to make it visible. A detail view can also reveal the natural appearance of the celestial body.

2. The planets and the sun are shown in a second map scale, which is independent of the orbits scale. With an enlargement factor of for instance 1000, all planets become visible and the surface of the Sun reaches almost the orbit of Jupiter.

In addition to the introduced dual scale approach, the Planet Tool provides further important features for high school education. Beside the current positions (up-to-date), the planet positions can be shown corresponding to an arbitrary date. An animation of the planets shows the different orbital velocities and motions along the elliptical orbits. The different eccentricities of the orbits become apparent due to a comparison with heliocentric circles.

Regarding the focus of this presentation, it can be stated that the application of two independent map scales allows an accurate and realistic cartographic depiction of the planets size in relation to their orbits and the sun. As 2-dimensional cartographic depictions always have known scales for all shown objects ${ }^{2}$, they still have a high value for educational purposes. The interactive modification of specific parameters by the user improves the comprehension of facts which are difficult to grasp, like for instance the true size of the planets in relation to their orbits.

\footnotetext{
${ }^{1}$ The Planet Tool online: https://www.planetentool.ch/.

${ }^{2}$ As opposed to 3-dimensional cartographic depictions.
} 